\title{
Does intensive glycemic control improve cardiovascular outcomes?
}

\author{
Merri Pendergrass
}

Until there is a cure for diabetes mellitus, the goal of treatment is to reduce symptoms and clinical complications. Microvascular and macrovascular complications are both strongly associated with hyperglycemia. Although good glucose control clearly reduces the risk of microvascular disease, it remains unclear whether this approach significantly reduces macrovascular disease.

Three recent randomized, clinical trials ACCORD (Action to Control Cardiovascular Risk in Diabetes Study Group [2008] N Engl $J$ Med 258: 2525), ADVANCE (ADVANCE Collaborative Group [2008] N Engl J Med 358: 2560) and VADT (Abraira C et al. [2008] Presented at the American Diabetes Association 68 ${ }^{\text {th }}$ Scientific Sessions: 2008 June 6-10, San Francisco, CA)-have shed light on this issue. These large, well-designed trials evaluated the effects of intensive glucose control (rather than a specific treatment regimen) on vascular outcomes in patients with type 2 diabetes and high cardiovascular risk. Although many features were shared, a number of differences might potentially have influenced the results of these trials, including median duration of follow-up (3.4-6.0 years), age at enrollment (60-66 years), baseline $\mathrm{HbA}_{1 \mathrm{c}}$ (7.2$9.4 \%)$, and target $\mathrm{HbA}_{1 \mathrm{c}}(<6.0-6.5 \%)$. At study end, the $\mathrm{HbA}_{1 \mathrm{c}}$ was $6.4-6.9 \%$ with intensive treatment and $7.0-8.4 \%$ with conventional treatment. The studies also differed with respect to use of specific diabetes medications, control of nonglycemic cardiovascular risk factors and use of cardioprotective agents, such as aspirin and statins. Nonetheless, the principal findings were consistent: near-normal glycemia did not have cardiovascular benefits. In ACCORD, near-normal glucose control was associated with an increased risk of death, the very outcome this trial was designed to prevent. Moreover, analyses from VADT indicated that the strongest predictor of heart attack or stroke was not hyperglycemia, but rather severe hypoglycemia.

...the results
of ACCORD,
ADVANCE
and VADT
highlight the
importance of
individualizing
glycemic goals

Several conclusions can be drawn from these three trials. First, intensive glucose control in patients with high cardiovascular risk should not be undertaken with a goal of improving cardiac outcomes over a 3-6 year period. Second, when normal glucose levels are targeted, there could potentially be adverse effects associated with the use of an increased number of drug classes, drug doses and/or regimen changes. In particular, the potential risks of hypoglycemia might outweigh treatment benefits.

These trials leave numerous questions unanswered. Whether intensive glucose lowering will have cardiovascular benefits in patients with low cardiac risk or over a longer duration of time is unknown. It is also unknown whether benefits will be observed in the years following completion of the trials. For example, cardiovascular benefits of glycemic control were not observed at the end of the 6.5-year intervention of the DCCT trial, but became apparent 11 years after the intervention was discontinued. Finally, both conventional and intensive treatment regimens were associated with fewer cardiovascular events than predicted. This observation suggests that modern riskreduction strategies, even when incompletely implemented, are indeed effective. As cardiovascular care is progressively optimized, it will probably become more difficult to demonstrate additional improvements in outcomes as a result of intensive glucose lowering.

In conclusion, the results of ACCORD, ADVANCE and VADT highlight the importance of individualizing glycemic goals. In order to achieve microvascular benefits, glucose levels should be maintained as close to normal as possible. This goal should be accomplished without causing excessive rates of hypoglycemia, other adverse effects or lifestyle burden. Lower goals are most appropriate for younger patients with minimal complications. Less intensive goals are indicated for patients with limited life expectancy, significant cardiovascular risk, or severe or frequent hypoglycemia. 